Pneumologe 2022 · 19:1-2

https://doi.org/10.1007/s10405-021-00424-y

Angenommen: 2. November 2021

(C) The Author(s), under exclusive licence to Springer Medizin Verlag GmbH, ein Teil von Springer Nature 2021

Redaktion

J. Gottlieb, Hannover

H. Worth, Fürth

\section{Nutzen und Risiken der Behandlung mit Sauerstoff}

\author{
Heinrich Worth ${ }^{1}$ Jens Gottlieb ${ }^{2}$ \\ ${ }^{1}$ Facharztforum Fürth, Fürth, Deutschland \\ ${ }^{2}$ Klinik für Pneumologie, Medizinische Hochschule Hannover, Hannover, Deutschland
}

Sauerstoff $\left(\mathrm{O}_{2}\right)$ ist ein Arzneimittel, das zur Behandlung einer Hypoxämie sowohl in der Akutmedizin als auch chronisch und im häuslichen Bereich eingesetzt wird. In der aktuellen S3-Leitlinie zum Einsatz von Sauerstoff in der Akuttherapie bei Erwachsenen und S2k-Leitlinie zur Langzeitsauerstofftherapie werden Nutzen und Risiken, Indikationsstellung, Durchführung und Überwachung der Behandlung mit Sauerstoff sowohl in der Notfallmedizin als auch für die Langzeitbehandlung mit Sauerstoff (LTOT, Long Term Oxygen Therapy) beschrieben.

\section{I) Sauerstoff wird in der Notfallmedizin zur Behandlung der akuten und der chronischen Hypoxämie eingesetzt}

$H$. Worth beschreibt die pathophysiologischen Grundlagen von Störungen der Oxygenation und die Möglichkeiten zur Erfassung einer Hypoxämie. Zur Charakterisierung der Schwere einer Hypoxämie $\left(\mathrm{S}_{\mathrm{a}} \mathrm{O}_{2}<90 \%, \mathrm{p}_{\mathrm{a}} \mathrm{O}_{2}<60 \mathrm{~mm} \mathrm{HG}\right)$ wird die einfach mittels Pulsoximetrie zu erfassende $\mathrm{S}_{\mathrm{p}} \mathrm{O}_{2}$ in der Notfallmedizin der aufwendigeren Messung des arteriellen Sauerstoffpartialdrucks $\left(\mathrm{p}_{\mathrm{a}} \mathrm{O}_{2}\right)$ vorgezogen, zumal für den in der Notfallmedizin häufig relevanten Bereich der $\mathrm{SO}_{2}$-Werte zwischen $40 \%$ und $90 \%$ eine nahezu lineare Beziehung zum $\mathrm{p}_{\mathrm{a}} \mathrm{O}_{2}$ besteht. Für die Differenzierung der Störungen des respiratorischen Gasaustausches sowie zur Indikationsstellung und Verlaufskontrolle einer LTOT ist die Blutgasanalyse hingegen unerlässlich. Angesichts der Gefährdung des Patienten sowohl durch Hypoxämie als auch durch Hyperoxämie sollte die $\mathrm{O}_{2}$-Behandlung in einem für das Krankheitsbild des Patienten definierten Zielbereich von $\mathrm{S}_{\mathrm{a}} \mathrm{O}_{2}$ bzw. $\mathrm{p}_{\mathrm{a}} \mathrm{O}_{2}$ erfolgen.

T. Fühner und J. Gottlieb beschreiben in Abhängigkeit vom Hyperkapnierisiko des Patienten und vom Status der Beatmung Zielbereiche der Sauerstoffsättigung in der Akutmedizin anhand der neuen Leitlinie. Etwa $25 \%$ der akutstationären Patienten weisen nämlich neben einer Hypoxämie auch eine Hyperkapnie auf. Für Patienten mit Hyperkapnierisiko soll der Zielbereich der $\mathrm{S}_{\mathrm{p}} \mathrm{O}_{2}$ zwischen $88 \%$ und $92 \%$ liegen, bei spontan atmenden Patienten ohne Hyperkapnierisiko zwischen 92\% und $96 \%$. Für beatmete Patienten wird eine arterielle Sättigung $\left(\mathrm{S}_{a} \mathrm{O}_{2}\right)$ zwischen $92 \%$ und $96 \%$ empfohlen. Diese Zielbereiche gelten bis auf wenige Ausnahmen (Wiederbelebungsmaßnahmen, ClusterKopfschmerz, Kohlenmonoxidvergiftung) für alle erwachsenen Patienten mit akuter Hypoxämie und unterscheiden sich nicht zwischen einzelnen Diagnosen. Die Autoren geben außerdem Empfehlungen zur Auswahl aus den vorhandenen Applikationssystemen unter den Gesichtspunkten Patientensicherheit und Patientenkomfort. Sie betonen die Notwendigkeit einer ärztlichen Verordnung, der Dokumentation, Überwachung und Reevaluation der Behandlung mit $\mathrm{O}_{2}$. Bei der Beendigung einer $\mathrm{O}_{2}$-Therapie soll auf das Risiko einer Hyperkapnie aufgrund einer Reboundhypoxämie geachtet werden.

J. Grensemann, M. Simon, C. Wachs und S. Kluge berichten über die High- 
Flow-Sauerstofftherapie („high flow nasal capsula" [HFNC]), die bei der Notwendigkeit von $\mathrm{O}_{2}$-Flussraten $>6 \mathrm{l} / \mathrm{min}$ zur Behandlung der hypoxämischen respiratorischen Insuffizienz unter ständiger Überwachung des Patienten zum Einsatz kommt. Bei dieser Therapieoption wird ein Sauerstoff-Luft Gas-Gemisch mit Flüssen zwischen 30 und $70 \mathrm{I} / \mathrm{min}$ appliziert. Die Technik kann auch während einer Bronchoskopie zur Oxygenierung, vor einer Intubation zur Präoxygenierung und nach Extubation zur Vermeidung einer Re-Intubation eingesetzt werden. Vorzüge des Verfahrens sind eine präzise Einstellung der inspiratorischen Sauerstofffraktion, die Erzeugung eines positiven endexspiratorischen Drucks, eine Verbesserung der mukoziliären Clearance sowie eine Reduktion der Atemarbeit. Da die Aerosolbildung durch Anwendung der HFNC bei guter Anpassung nicht erhöht ist, kann diese auch bei Patienten mit COVID19 eingesetzt werden. Bei respiratorischer Insuffizienz mit Hyperkapnie sollte hingegen eine nichtinvasive Maskenatmung (NIV) bevorzugt werden.

Nach P. Haidl soll durch die Langzeitsauerstofftherapie (LTOT) eine Senkung der Sterblichkeit und Verbesserung der Leistungsfähigkeit von Patienten mit chronischen Lungenkrankheiten erreicht werden. Voraussetzung für die LTOT sind in stabiler Krankheitssituation 2 kapilläre (arterielle) Blutgasanalysen im Abstand von 3 Wochen mit Nachweis einer Hypoxämie mit einem $\mathrm{p}_{\mathrm{a}} \mathrm{O}_{2} \leq 55 \mathrm{~mm} \mathrm{Hg}$. Liegt der $\mathrm{p}_{\mathrm{a}} \mathrm{O}_{2}$ zwischen 55 und $60 \mathrm{~mm} \mathrm{Hg}$, sollten zusätzlich ein Cor pulmonale oder eine Polyglobulie nachweisbar sein. Eine $\mathrm{O}_{2}$-Sättigung $>89 \%$ und ein $\mathrm{p}_{\mathrm{a}} \mathrm{O}_{2}>60 \mathrm{~mm} \mathrm{Hg}$ sind keine Indikation zur LTOT. Die nach einer stationären Behandlung wegen einer Exazerbation verordnete Sauerstoffversorgung ist innerhalb von 3 Monaten nach der Entlassung zu überprüfen und ggf. zu beenden. Der Einsatz der LTOT bei fibrosierenden Lungenerkrankungen und chronischer Herzinsuffizienz erfolgt aufgrund der geringen Datenlage analog zu den Kriterien bei COPD ("chronic obstructive pulmonary disease").

J. Geiseler und M. Basler informieren über die Möglichkeiten der $\mathrm{O}_{2}$-Versorgung zu Hause, wobei die Art der Versorgung unter den Gesichtspunkten Zweckmäßigkeit,
Effizienz und Wirtschaftlichkeit von den Kostenträgern getroffen wird. Der Beitrag gibt auch Auskunft über formale und rechtliche Aspekte der Verordnung der Sauerstoffversorgung mit ihren spezifischen Vorzügen und Nachteilen, um den Ärzten Entscheidungshilfen an die Hand zu geben. Die immer häufiger verordneten mobilen Konzentratoren müssen, da die Sauerstoffabgabe überwiegend im Demand-Modus erfolgt, immer individuell bei jedem Patienten getestet werden. Schließlich werden praktische Aspekte der LTOT, wie z.B. die Befeuchtung, diskutiert.

Wir hoffen, mit den 5 Beiträgen einen für die klinische Arbeit von Internisten, Notfall- und Intensivmedizinern, Pneumologen und Pflegenden nützlichen und aktuellen Überblick über den Einsatz von Sauerstoff sowohl in der Intensiv- und Notfallmedizin als auch für die Langzeitbehandlung zu geben.

Prof. Dr. Heinrich Worth

Prof. Dr. Jens Gottlieb

\section{Korrespondenzadresse}

\section{Prof. Dr. Heinrich Worth}

Facharztforum Fürth

Bahnhofplatz 6, 90762 Fürth, Deutschland

h.worth49@gmail.com

Interessenkonflikt. H. Worth und J. Gottlieb geben an, dass kein Interessenkonflikt besteht. 